\title{
Residual Stress Measurements with Laser Speckle Correlation Interferometry and Local Heat Treating (U)
}

by

M. J. Pechersky

Westinghouse Savannah River Company

Savannah River Site

Aiken, South Carolina 29808

R. F. Miller

Westinghouse Savannah River Company

SC USA

C. S. Vikram

University of Alabama

AL USA

A document prepared for ELECTRONIC IMAGING:SCIENCE AND TECHNOLOGY at San Jose from 02/05/95 02/10/95.

DOE Contract No. DE-AC09-89SR18035

This paper was prepared in connection with work done under the above contract number with the U.S.

Department of Energy. By acceptance of this paper, the publisher and/or recipient acknowledges the U.S. Government's right to retain a nonexclusive, royalty-free license in and to any copyright covering this paper, along with the right to reproduce and to authorize others to reproduce all or part of the copyrighted paper. 


\section{DISCLAIMER}

This report was prepared as an account of work sponsored by an agency of the United States Government. Neither the United States Government nor any agency thereof, nor any of their employees, makes any warranty, express or implied, or assumes any legal liability or responsibility for the accuracy, completeness, or usefulness of any information, apparatus, product, or process disclosed, or represents that its use would not infringe privately owned rights. Reference herein to any specific commercial product, process, or service by trade name, trademark, manufacturer, or otherwise does not necessarily constitute or imply its endorsement, recommendation, or favoring by the United States Government or any agency thereof. The views and opinions of authors expressed herein do not necessarily state or reflect those of the United States ${ }^{-}$Government or any agency thereof.

This report has been reproduced directly from the best available copy.

Available to DOE and DOE contractors from the Office of Scientific and Technical Information. P. O. Box 62, Oak Ridge. TN 37831; prices available from (615) $576-8401$.

Available to the public from the National Technical Information Service, U. S. Deparment of Commerce, 5285 Port Royal Rd., Springfield, VA 22161 


\section{DISCLAIMER}

Portions of this document may be illegible in electronic image products. Images are produced from the best available original document. 


\title{
Residual stress measurements with laser speckle correlation interferometry and local heat treating
}

\author{
M. J. Pechersky, R. F. Miller \\ Westinghouse Savannah River Company, Savannah River Technology Center \\ Aiken, South Carolina 29802 \\ C. S. Vikram \\ University of Alabama in Huntsville, Center for Applied Optics \\ Huntsville, Alabama 35899
}

\begin{abstract}
A new experimental technique has been devised to measure residual stresses in ductile materials with a combination of laser speckle pattern interferometry and spot heating. The speckle pattern interferometer measures in-plane deformations while the heating provides for very localized stress relief. The residual stresses are determined by the amount of strain that is measured subsequent to the heating and cool-down of the region being interrogated. A simple lumped parameter model is presented to provide a description of the method. This description is followed by presentation of the results of finite element analyses and experimental results with uniaxial test specimens. Excellent agreement between the experiments and the computer analyses were obtained.
\end{abstract}

\section{INTRODUCTION}

Residual stresses can occur in many engineering structures such as airplanes and nuclear reactors and can sometimes lead to premature failures. Residual stresses may be defined as "those stresses existing without (and generally prior to) the application of any intended, or unintended external loads ${ }^{1} . "$ Residual stresses are produced by many material processing and joining operations such as; welding, machining, rolling and others. In many instances tensile residual stresses can result in fatigue failures and promote corrosion. On the other hand, compressive residual stresses are sometimes purposely induced in materials by shot peening, water peening or rolling to help resist fatigue by reducing the potential for crack growth. Interest in either the measurement or formation of residual stresses exists in many industries. These industries include: manufactures of piping and pressure vessels such as oil refineries, chemical plants and power plants; the material processing industry, the transportation industry and the electronics industry.

There are two commonly used methods by which residual stresses are currently measured. One method is known as blind hole drilling 2,3 and the other method is X-ray diffraction ${ }^{4}$, or XRD. Neutron diffraction is a third method, however it is restricted to the laboratory because this method requires an intense neutron source such as a nuclear reactor. Other techniques such as Barkhausen Noise, Ultrasonics and others have been ${ }^{5}$ and are being developed.

We are developing a new experimental technique to measure residual stresses with a combination of laser speckle pattern interferometry and spot heating. The technique is based on the well known idea that when stressed material is heated, the stress may be relieved to a certain degree based on the time and temperature of the heat treating process and the stress-strain characteristics of the material. The ultimate measurement technique is to be done by heating a small spot in the region of interest with an infrared laser and determining the net plastic flow with an electronic speckle pattem interferometer. If one knows the material properties and the heating/cooling cycle, one can deduce the residual stresses. This approach is being developed because it is totally nondestructive and has the potential to be an easily applied in situ during a field measurement.

The process to determine the residual stress can be envisioned with the simple lumped parameter example, which is discussed next. This simple lumped parameter model only crudely represents the actual process, but in addition to providing this physical description of the process, it defines what material properties and other parameters are required for a measurement. 


\section{LUMPED PARAMETER MODEL}

Consider a spring of length, $L$ which is initially relaxed and at a temperature $T_{L}$. A displacement of unknown amount, $x_{1}$ is applied to the spring and the ends are then fixed. Thus, the value of the applied force is also unknown. The displacement and force are related through Hooke's law by:

$$
\mathrm{F}_{1}=\mathrm{k}_{\mathrm{L}} \cdot \mathrm{x}_{1},
$$

where $\mathrm{k}_{\mathrm{L}}$ is the spring constant of the spring at temperature, $\mathrm{T}_{\mathrm{L}}$. The strain energy stored in the spring can be expressed as:

$$
\mathrm{U}_{1}=\frac{1}{2}\left(\frac{\mathrm{F}_{1}^{2}}{\mathrm{~kL}_{\mathrm{L}}}\right)
$$

In this example the spring is assumed to be in tension and, of course, the deformation is assumed to be elastic. This initial state is shown as point $I$ in the force-displacement diagram shown in Figure 1 . If the spring is subsequently heated the force will relax due to thermal expansion and plastic flow. The plastic flow results from the reduced yield stress of the material at the elevated temperature, $T_{\mathrm{H}}$. The state of the spring after it has been heated is shown as point II on Figure 1. Point II is located on a forcedisplacement diagram corresponding to the higher temperature, $T_{\mathrm{H}}$. The force-displacement curve is shifted by an amount, $x_{\mathrm{th}}$ to take into account thermal expansion. Since the ends of the spring are fixed, the total displacement, $x_{1}$ doesn't change but it may now be decomposed into three "virtual" components; the thermal expansion component given by:

$$
\mathrm{x}_{\mathrm{th}}=\alpha\left(\mathrm{T}_{\mathrm{H}}-\mathrm{T}_{\mathrm{L}}\right) \mathrm{L}
$$

the elastic component given by:

$$
\mathrm{XHE}_{\mathrm{HE}}=\frac{\mathrm{F}_{\mathrm{HY}}}{\mathrm{k}_{\mathrm{H}}}
$$

and the plastic component at the elevated temperature, $\mathrm{x}_{\mathrm{pH}} . \mathrm{F}_{\mathrm{HY}}$, is the force at which the spring yields as indicated in Figure 1. The material is assumed to be perfectly plastic. When the spring is allowed to cool the thermal expansion is reversed but the plastic deformation remains so the force on the spring is:

$$
F_{3}=k_{L}\left(x_{L}-x_{p}\right)
$$

Point III, is on yet a third force-displacement curve, which is now shifted due to the plastic flow. In this third state the spring constant reverts back to $\mathrm{k}_{\mathrm{L}}$. The energy stored in the spring is:

$$
\mathrm{U}_{3}=\frac{1}{2}\left(\frac{\mathrm{F}_{3}^{2}}{\mathrm{k}_{\mathrm{L}}}\right)
$$

The amount of elastic energy dissipated as a result of the stress relief is:

$$
U_{d}=\left\{\frac{F_{1}}{k_{L}}-\left(\frac{F_{H Y}}{k_{H}}+\alpha\left[T_{H}-T_{L}\right]\right)\right\} F_{H Y}
$$

Now, the initial stored energy in the spring must be equal to the final stored energy plus the energy dissipated due to the plastic flow, or:

$$
\mathrm{U}_{1}=\mathrm{U}_{\mathrm{d}}+\mathrm{U}_{3}
$$


which leads to the following equation for $F_{1}$ (the unknown force) in terms of the physical parameters of the spring and the amount of plastic flow, i.e.:

$$
F_{1}=\frac{\left\{\frac{k_{L} \cdot x_{P}^{2}}{2}-\frac{F_{Y H}^{2}}{k_{H}}-\alpha\left(T_{H}-T_{L}\right) F_{Y H}\right\}}{\left(x_{P}-\frac{F_{Y H}}{k_{L}}\right)}
$$

If one assumes that the spring is a constant cross section bar; Equation 9 can be recast into an equation involving stress and strain as follows:

$$
\sigma=\frac{\left\{\left(\frac{E_{L}}{2}\right) \varepsilon_{a}^{2}-\frac{\sigma_{Y}^{2}}{E_{H}}-\left(\sigma_{Y} \cdot \alpha \cdot \Delta T\right)\right\}}{\left(\varepsilon_{a}-\frac{\sigma_{y}}{E_{L}}\right)}
$$

Where: $\quad \sigma=$ the value of the unknown tensile or compressive stress being measured,

$E_{\mathrm{L}}=$ the value of Young's Modulus prior to laser heating,

$\sigma_{Y}=$ the yield stress at the elevated temperature, $T_{H}$

$\mathrm{E}_{\mathrm{H}}=$ the value of Young's Modulus at the elevated temperature,

$\alpha=$ the coefficient of thermal expansion of the material; averaged over the range of temperatures expected, $\Delta T=$ the temperature rise due to the laser heating, $\left(T_{H}-T_{L}\right)$

and:

$$
\varepsilon_{\mathrm{a}}=\text { the "virtual" in-plane strain resulting from the stress relaxation. }
$$

Thus, if the material properties are known one need only determine this value of the strain, $\varepsilon_{\mathrm{a}}$. Since in this example the total displacement was assumed to be fixed one could not do a macroscopic displacement measurement. (Note that $\varepsilon_{\mathrm{a}}$ could, in principal, be measured with XRD.)

Now, imagine instead, that the same process is repeated, but now, the heated spring is placed in series with another spring so the total displacement of the series combination is fixed. Furthermore, assume that the unheated spring undergoes only elastic deformations and that the thermal and force relaxation processes can be superposed. Then the heated and the unheated spring would deform in equal and opposite directions. In this case the unknown force or stress can be determined by the final displacement of the point connecting the two springs or alternatively the net strain in the unheated spring. This latter approach is roughly analogous to the spot heating techniques that we are developing.

The optical method to determine the in-plane strain relief, $\varepsilon_{\mathrm{a}}$ is based on a scheme presented by Jones and Wykes ${ }^{6}$. With this type of interferometer the object is illuminated by two beams at plus and minus $\theta$ from the surface normal. For the one dimensional situation described here the strain is given by:

$$
\varepsilon_{a}=\frac{\lambda}{(2 \cdot \Delta x \cdot \sin \theta)}
$$

Where: $\quad \lambda=$ the wavelength of the laser light for the speckle interferometer,

$$
\Delta \mathrm{x}=\text { the measured fringe spacing after performing the image subtraction, }
$$

$\theta=$ the angle of incidence of the interferometer laser beams.

and the direction of the strain is along the projection of the illuminating beams on the surface of the object. The details of the interferometer employed in our experiments will be discussed later. To first order this type of interferometer is insensitive to changes in the elevation or distance of the surface relative to the interferometer optics. This is different than holographic methods in which there is always some sensitivity to the out-of-plane deformations. These out-of-plane deformations are a second order effect and therefore not as pronounced as the in-plane strains. Holographic techniques have, however been used successfully in the past in conjunction with blind hole drilling methods? 


\section{COMPUTER SIMULATIONS}

While the previous discussion defines the global parameters of the measurement a detailed analysis is required. The temporal and spatial characteristics of the heat required treatment for the measurement must be specified. In addition, the detailed thermomechanical response of the specimen under test is needed so that we can compare this detailed response to the results of an interferometric measurement. The $\mathrm{ABAQUS}^{8}$ finite element computer software was employed to perform these analyses. The results of these analyses are presented in the following discussion.

The heating process must be accomplished so that it does not create a nonlinear response in the material, other than the stress relaxation process described above. That is, if the material is heated too much or too fast the local thermal expansion can create plastic compressive strains. This will confound the measurement. In theory, the measurement could still be accomplished, but the analysis becomes so complex as to make the technique impractical. Prior to performing the finite element analyses (FEA), a theoretical analysis was performed to estimate the temperature at which this unwanted nonlinearity creeps into the measurement. The analysis was based on the sudden heating of a large, unloaded, 304 stainless steel plate. The results of this analysis are shown in Figure 2. Figure 2 contains a plot of the increasing compressive stresses generated as a function of temperature and the corresponding decrease in yield stress. Once the thermally induced stresses exceed the yield stress, the plate starts to plastically deform. In this analysis, a temperature rise of only 50 degrees Celsius is required. This is a very imprecise estimate, but it points out the order of magnitude of the allowable temperature increase, and the need for further analysis.

As a result, a two dimensional finite element analysis was performed with more realistic boundary conditions. The heating was chosen to simulate a laser beam with a Gaussian intensity profile. For this part of the analysis, we were only interested in determining the maximum amount of heat addition and the maximum heating rates that could be applied without inducing plastic yielding due to thermal expansion. Therefore, a simple physical model was chosen for the specimen geometry. The model is that of two dimensional, 304 Stainless Steel disk, which was heated at its center. A vertical slice through this disk with the element geometry used in the calculations is shown in Figure 3. The heat source was assumed to be a laser beam with a Gaussian intensity profile. The disk was assumed to be stress free prior to the laser heating.

Several spot sizes, heating rates, heat fluxes and pulse widths were used as input parameters in the analysis. The only clear functional relationship between these parameters, and when yielding occurred was the maximum temperature rise. While the larger spot size and longer heating pulse durations resulted in some plastic deformation, this effect was small compared to the effect of peak temperature. A summary of the results is shown in Figure 4. This is a plot of Maximum Equivalent Plastic Strain versus Peak Temperature rise in the specimen. The Maximum Equivalent Plastic Strain is related to the mechanical energy that is dissipated due to the plastic deformation. The data points include all of the different spot sizes, heat fluxes, pulse shapes and pulse widths analyzed. The final heating cycle chosen based on this analysis was a $2 \mathrm{~mm}$ diameter spot with a peak intensity of $400 \mathrm{~W} / \mathrm{cm}^{2}$. The heating pulse was a triangular in shape starting at no power, increasing to full power in 2.5 seconds and then falling off to zero in another 2.5 seconds. The maximum power of a laser beam to supply this heating would be about 12.5 watts. Naturally, the laser power would have to be increased to take into account reflection.

The final thermo-mechanical analysis was performed to simulate the experiments. The test samples were 304 Stainless Steel, ASTM E8-91 Sheet Type, tensile specimens. In the analysis, the specimens were assumed to be at various levels of uniform axial tension prior to the application of the heat. The protocol described in the preceding paragraph was used for the heat input. The finite element model simulated the constant cross section part of the specimen. The geometry used in the analysis is shown in Figure 5. Figures 6,7 and 8 show the calculated results for stresses equal to 80,90 and 98 percent of the room temperature yield stress. These figures show the net longitudinal displacement on the surface of the material as a function of position in the region surrounding the heated spot. These are the displacements after the specimen has completely cooled and therefore correspond to point III in the simple spring model discussed above. As in the spring model, the total length of the specimen was assumed to be fixed. The regions on the figures show areas of constant displacement, according to the respective gray scales next to the main part of each figure. Since these are displacements and not strains, they get larger, the further they are away from the heated spot. 
Figures 6,7 and 8 cover only for one quadrant, since there is mirror symmetry around both the lateral and longitudinal sample axes. The maximum displacements are about 6 micrometers for the highest residual stress case and about 0.6 micrometers for the lowest residual stress case. These deformations can easily be measured with the type of speckle interferometer mentioned earlier. The experiments will be described and the results of this analysis and the experimental results will be compared in the following discussion.

\section{DESCRIPTION OF EXPERIMENTS}

A series of experiments were performed to demonstrate the validity of the approach described in the first part of this paper. The tests were performed by preloading the test specimens in uniaxial tension to a given stress level and then fixing the ends of the specimen. In this way the experimental conditions matched the conditions imposed by the computer simulation. In a "real" situation it is arguable as to whether the stress or the displacement is held constant. As the measurement procedure is developed this issue will need to be addressed. The heating was supplied by contact resistance rather than laser heating. Ultimately, laser heating would be the approach of choice to do these residual stress measurements. As mentioned earlier a relatively low power carbon dioxide laser would probably be most suitable. For the experiments reported here, however, the heating was accomplished by passing current through a graphite tip into the test specimen. This method proved to be workable and avoided the need to acquire a laser until the validity of this method of stress measurement could be verified. As will be seen, the experimental results presented in the following discussion and the computer simulations were in excellent agreement.

\subsection{Description of the apparatus}

Figure 9 shows a schematic of the load frame and heating apparatus used in these experiments. The preload was determined by a load cell that was fixed between the bottom grip (which is fixed) and the frame of the tensile tester. A dial gauge was positioned on the top of the load frame to measure the displacement of the top grip, which was the movable grip. The top grip was driven through lead screws to apply the desired load. The friction on the motor and lead screws was sufficient to clamp the end of the specimen as born out by the dial gauge readings. Current for the electrical heating was supplied by a programmable power supply. The current wave form and heating cycle were chosen to mimic the thermal cycle used in the computer simulations. This was done by adjusting current pulse in magnitude and time so that the peak temperature on the back side of the specimen was the same as that in the FEA model. The heating element ultimately selected for the demonstration experiments had a $2 \mathrm{~mm}$ diameter soft graphite tip. The temperature was measured with a thermocouple pressed to the back side of the specimen directly opposite of the contact point of the heating element. Care was taken to insure that the contact pressure of the thermocouple did not influence the measurement.

A schematic of the Electronic Speckle Pattern Interferometer (ESPI) optics is shown in Figure 10. In this setup a single beam expander/collimator was used. The collimated beam is split into two beams which illuminate the same spot on the test specimen. The angles of incidence were plus and minus 45 degrees. The illuminated spot was imaged onto a monochrome CCD camera as shown in Figure 11. The initial image is obtained prior to heating and stored in the memory of the micro-computer. Heat is applied and then specimen is allowed to cool. After the cool-down the second image is acquired. The images are subtracted and the resulting fringe pattern is displayed on a monochrome monitor. Prior to printing, the fringe pattern was low pass filtered and the contrast enhanced. The resulting fringe patterns are of course sensitive only to the longitudinal displacement on the surface of the specimen. .

\section{EXPERIMENTAL RESULTS AND COMPUTER MODEL COMPARISON}

The specimens used for the demonstration experiments were constructed according to ASTM Standard ${ }^{9}$ E8-91 for sheet type specimens. The constant cross section part of the 8 inch long specimen was 2 inches in length and 0.5 inches wide. The thickness of the specimen was 0.1 inch. The heating current was a square pulse with a duration of 3 seconds and a current of fifty amps. This current pulse and the resulting temperature on the back side of the 304 stainless steel specimen is shown in Figure 12. This heating cycle was used in all of the demonstration experiments. The required temperature rise on the back of the specimen as defined by the computer model was 47.4 degrees Celsius. The corresponding computed temperature rise at the heated surface was 187.8 degrees Celsius. The model also predicts about a one second lag between the heated surface and the back surface. This time lag is consistent with the lag between the current pulse and the temperature shown in Figure 12. 
Figure 13 shows the experimental results for the case when the preload was $80 \%$ of the nominal yield stress of the material. The longitudinal displacement, $\mathrm{u}_{\mathrm{x}}$ can be computed by integrating Equation 11 which can be written simply as:

$$
\mathrm{u}_{\mathrm{x}}=\frac{\mathrm{n} \cdot \lambda}{2 \cdot \sin \theta}
$$

where $\mathrm{n}$ is the order of the dark fringes. In this case as in the next two figures the center dark fringe is the zero order fringe. The intersection of the center of this zero order fringe and horizontal line in the figure is the spot at which the heating took place. It can be seen from the fringe pattern that this point is an axis of symmetry. The fringe spacing can be estimated by remembering that the width of the specimen was one half of one inch. The displacement obtained for this fringe pattern is 0.28 micrometers. The displacement obtained from the computer simulations was 0.275 micrometers. Thus the results of this measurement and the computations are in very good agreement. On the other hand one must admit that with the small displacement, in this experiment the interpretation of the fringe pattern is somewhat subjective. This is not so much the case for the higher stress levels. Figures 14 and 15 are similar to Figure 13 except they are for $90 \%$ and $98 \%$ of the yield stress, respectively. As the stress levels are raised one expects the amount of stress relaxation to increase. This intuitive result can also be easily deduced from Equation 10 . As the displacements increase, the number of fringes increases and the interpretation becomes much more concrete. As can be seen in Figure 14, the calculations and experimental results agree to within about 5\%. For Figure 16 the agreement is within about $6 \%$. These results are, of course, very encouraging.

\section{SUMMARY AND CONCLUDING REMARKS}

The work reported in this paper had as its objective: to demonstrate the "scientific" feasibility of determining residual and applied stresses by Electronic Speckle Pattern Interferometry (ESPI) and local annealing. We anticipate in the end that the heat for the annealing process will be supplied by a modest power $\mathrm{CO}_{2}$ laser. To this end we have developed a simple theoretical lumped parameter model to explain the process and to determine the essential features of the process. From this lumped parameter model, it can be seen that the yield stress of the material must be known as well as the more easily available parameters such as the Young's moduli at the high and low temperatures and the thermal expansion coefficient.

Computer simulations were subsequently performed and compared with experimental results'using an ESPI of our own construction. Excellent agreement between the computer model and the experiments was obtained. To obtain a complete description of the stress state by this measurement technique, the stress relaxation will have to be measured in three directions. This requirement is the same for blind hole drilling. To make this technique practical, several technical issues need to be addressed. One of the largest is the uncertainty in the yield stress of the material. Handbook values of the yield stress are, of course, commonly available. However, since the amount of cold work that the material has undergone may not be known, an in situ measurement may be required. It may be possible to estimate the yield stress with a hemispherical indenter, but more research on this subject is required. The remaining issues are less fundamental but still require additional work. These issues include: front surface temperature measurement; vibration isolation (for in situ measurements); heating cycles for depth profiling of the stresses; automated fringe analysis techniques, and finally the generation of a widely accepted measurement standard. We are presently working on resolving these issues.

We would like to reiterate, that while this method may seem to be unduly complex, in the final embodiment it is not expected to be. In our opinion, many of the operational and technical complexities can be easily handled. Our current work is geared towards doing an "engineering" feasibility demonstration in which this will become clear.

\section{ACKNOWLEDGMENTS}

We would like to thank Mr. Darell Englehaupt who lead the development of the load frame and heating element used in the exṕeriments.

The information contained in this article was developed during the course of work under Contract No. De-AC09-89SR18035 with the U.S. Department of Energy. By acceptance of this paper, the publisher and/or recipient acknowledges the U.S. Government's right to retain a non-exclusive, royalty-free license in and to any copyright covering this paper along with the right to reproduce, and to authorize others to reproduce all or part of the copyrighted paper. 
Figure 1. Force-displacement diagram for the simple spring model. The energy dissipated due to plastic flow is represented by the shaded area. The net amount of plastic flow, $x_{\mathrm{p}}$, is determined by this energy loss.

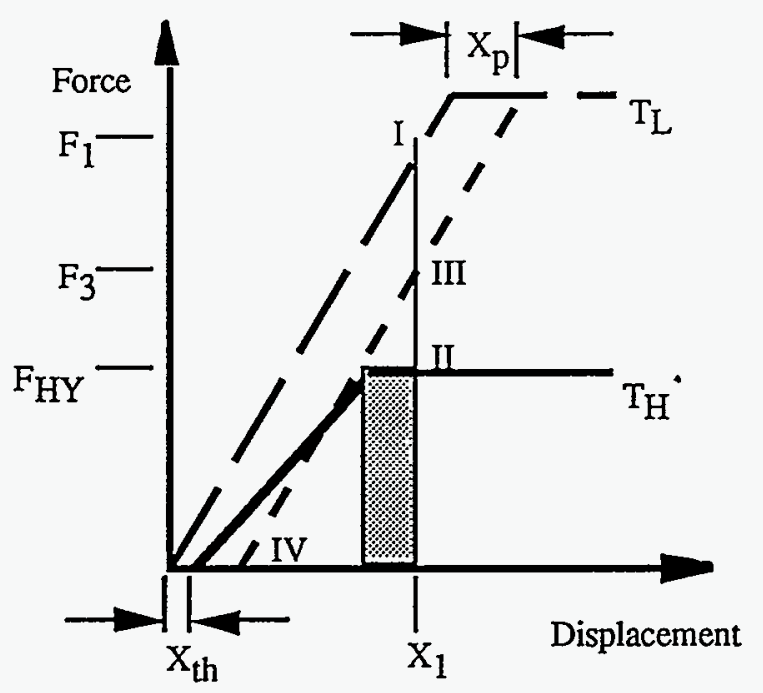

Figure 2. Thermal stresses and yield stresses generated in a semi-infinite half space. Once the stress levels exceed the yield stress, the measurement technique described in this paper becomes invalid. The laser heating process must be chosen to prevent this from happening.

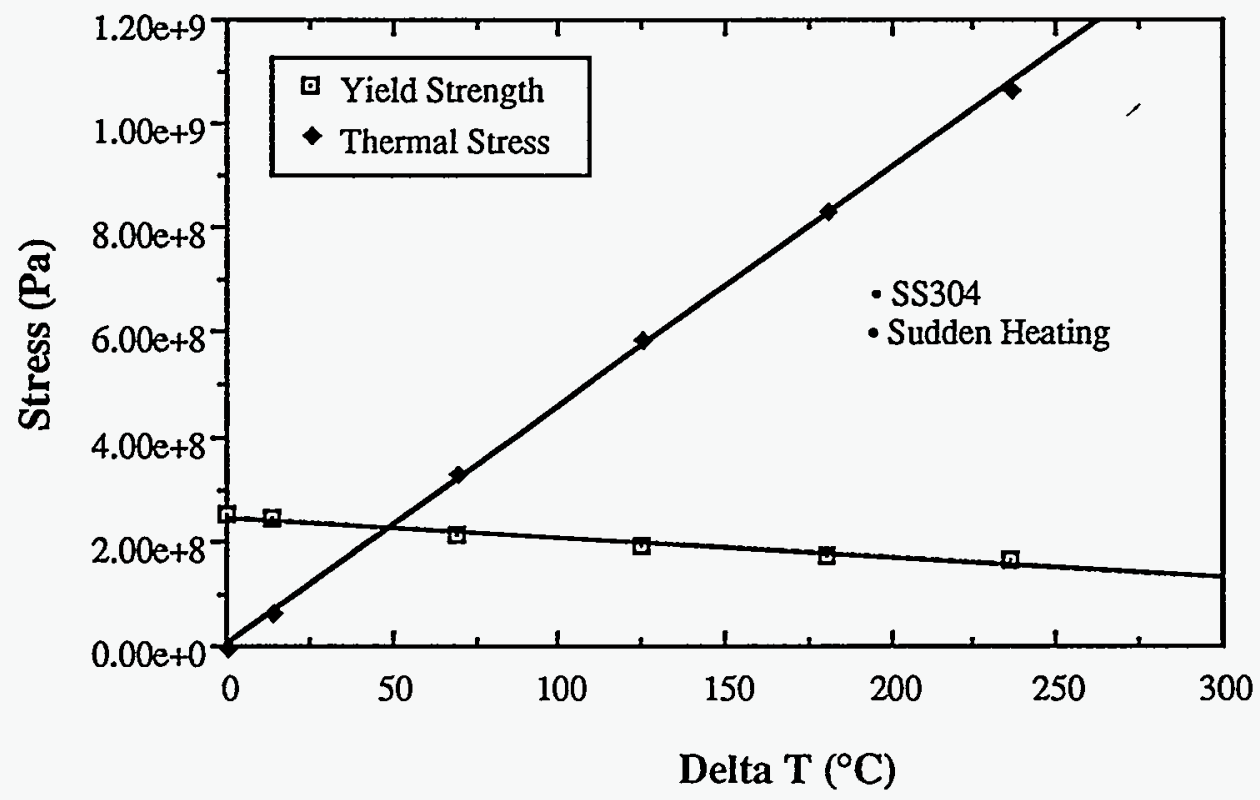


Figure 3. Two-dimensional, axi-symmetric disk, finite element model. The appropriate heating protocol to provide sufficient plastic strain relaxation, while avoiding thermally induced plastic strain was computed with this geometric model. The disk was assumed to be heated at its center, on the top surface, with an a Gaussian laser beam. Thermal-mechanical variations from the heated surface into the depth of the disk are included in the analysis.

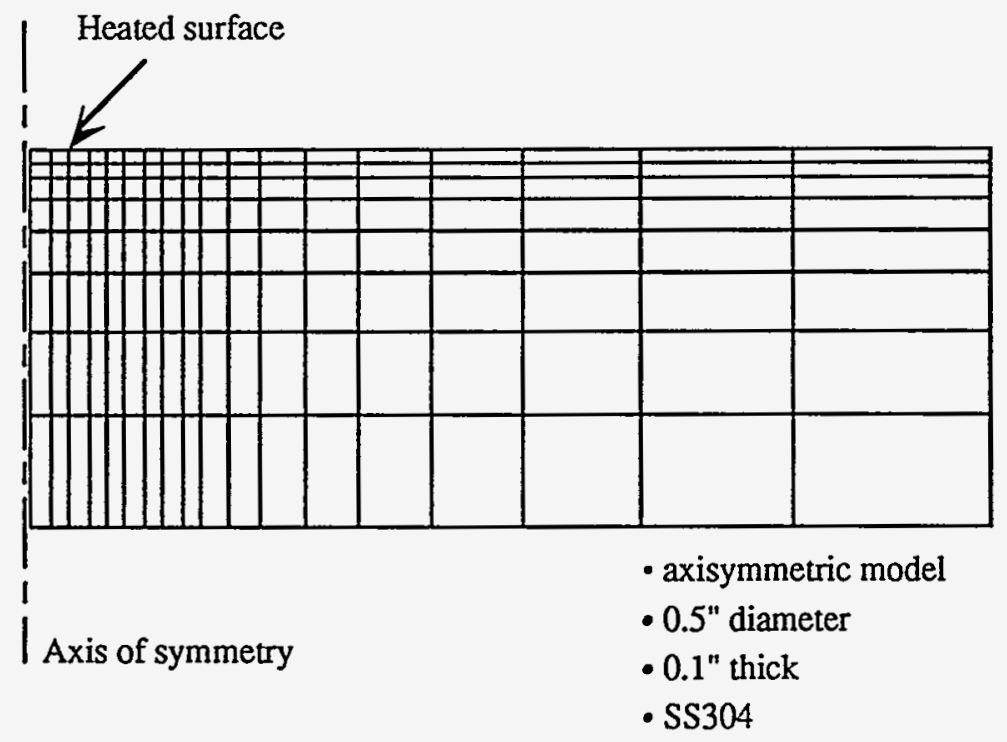

Figure 4. Correlation between temperature rise and thermally induced plastic strain. The heating protocol must be chosen such the there is negligible thermally induced plastic strain. Based on a range of calculations the heating protocol that was used in the full three dimensional finite element analysis was chosen using the disk model.

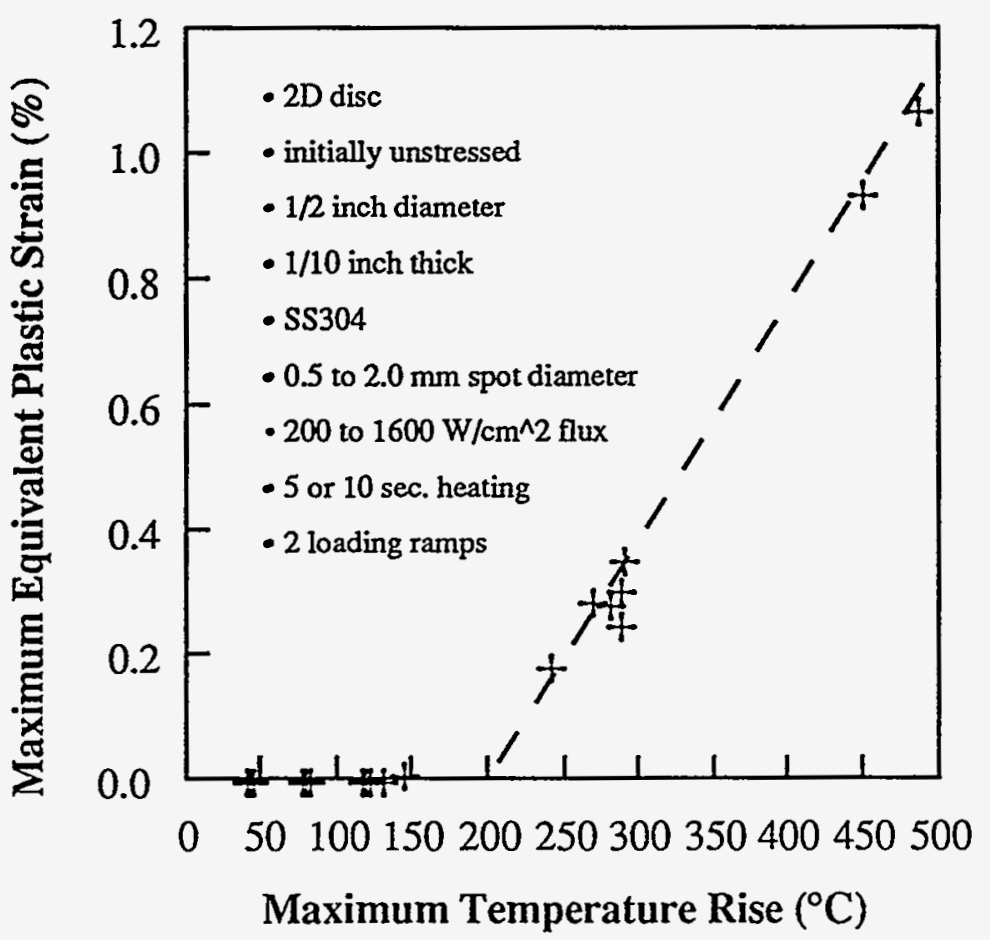


Figure 5. Specimen geometry used for finite element analysis simulations of the experiments. The simulations were based on a full three dimensional model. Heating was applied to one surface, at the centroid of the test specimen. Thus, symmetry could be taken advantage of since, only the upper right quadrant of the figure had to be modeled.

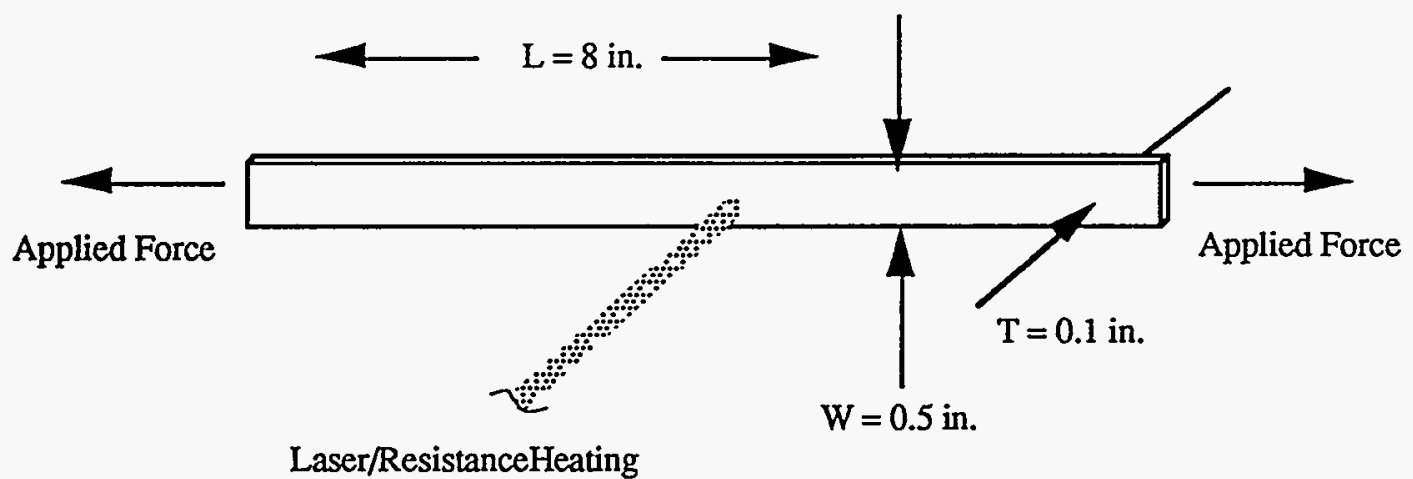

Figure 6. Displacements After heating and cooling for a load of $98 \%$ of the yield stress at room temperature. The gray scale on the right indicates the total axial displacement. The displacements are shown for a small region surrounding the heated spot. Since quarter symmetry obtains, only the upper right hand quadrant is shown in.

Y DISPLACEMENT AFTER HEATING FOR 98\% PRESTRAIN

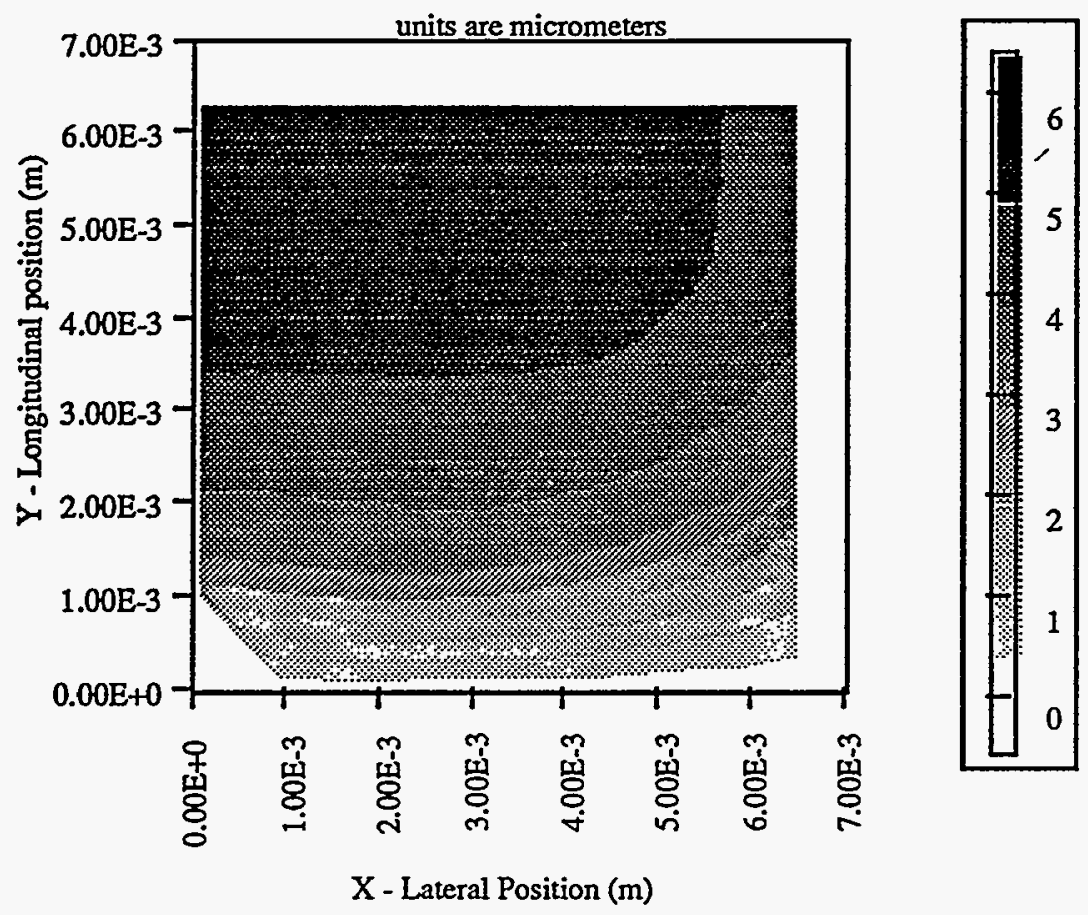


Figure 7. Displacements After heating and cooling for a load of $90 \%$ of the yield stress at room temperature. Maximum axial displacements are only about one third of those in $98 \%$ yield stress case.

\section{Y DISPLACEMENT AFTER HEATING FOR 90\% PRESTRAIN}

units are micrometers
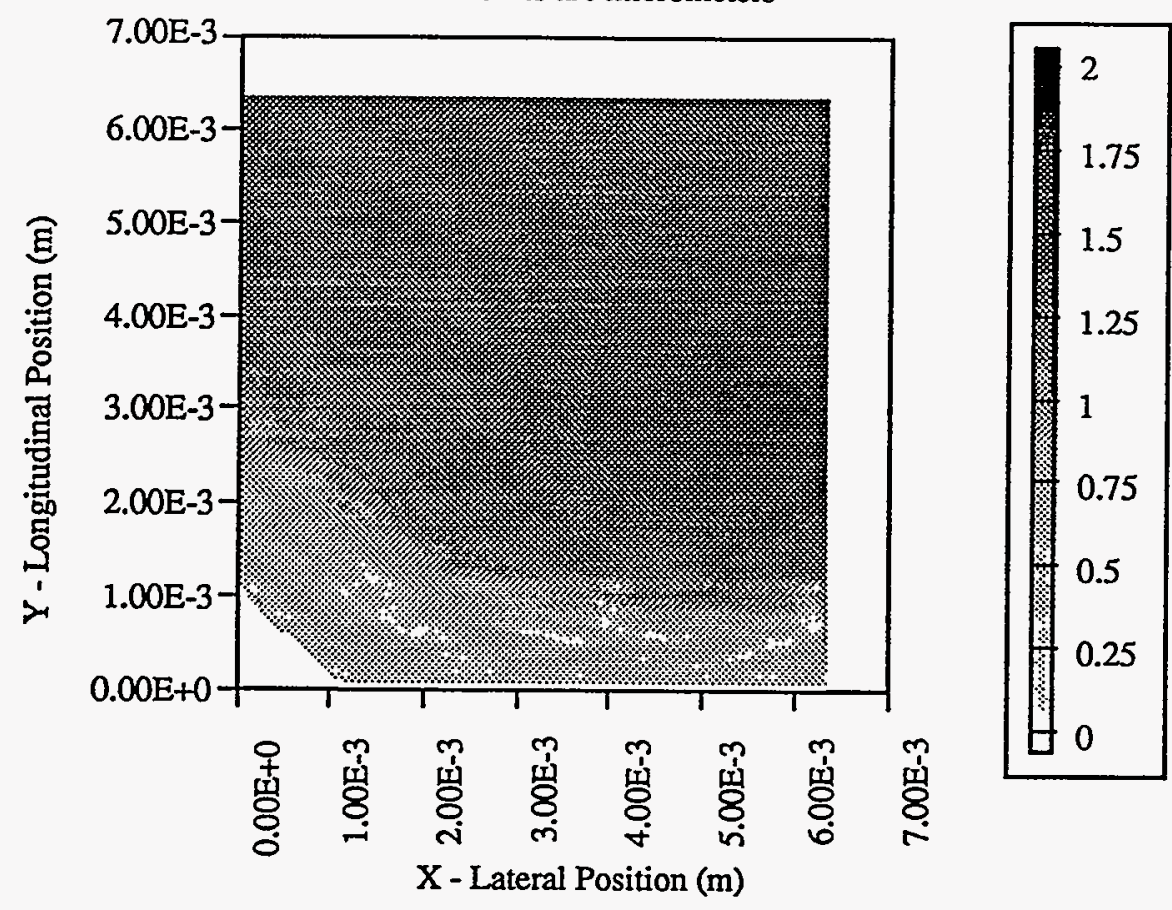

Figure 8. Displacements After heating and cooling for a load of $80 \%$ of the yield stress at room temperature. Maximum axial displacements are only about one tenth of those in $98 \%$ yield stress case.

\section{Y DISPLACEMENT AFTER HEATING FOR 80\% PRESTRAIN}

units are micrometers
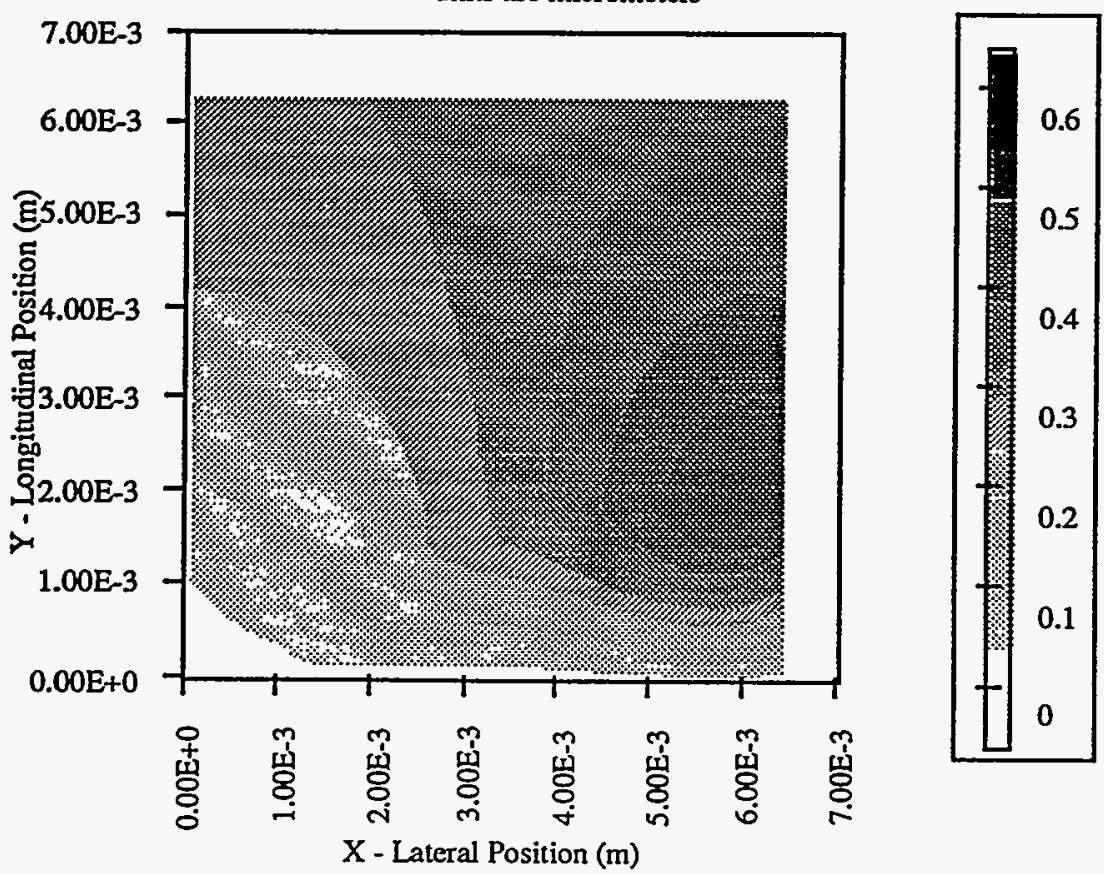
Figure 9. Schematic of the load frame and spot heating system. A small load frame preloads the test specimen which is then heated by contact resistance heating. A dial gauge was placed on the load frame to insure that the ends of the specimen remained fixed during the experimentation. The fixed end conditions were assumed in the computer simulations.

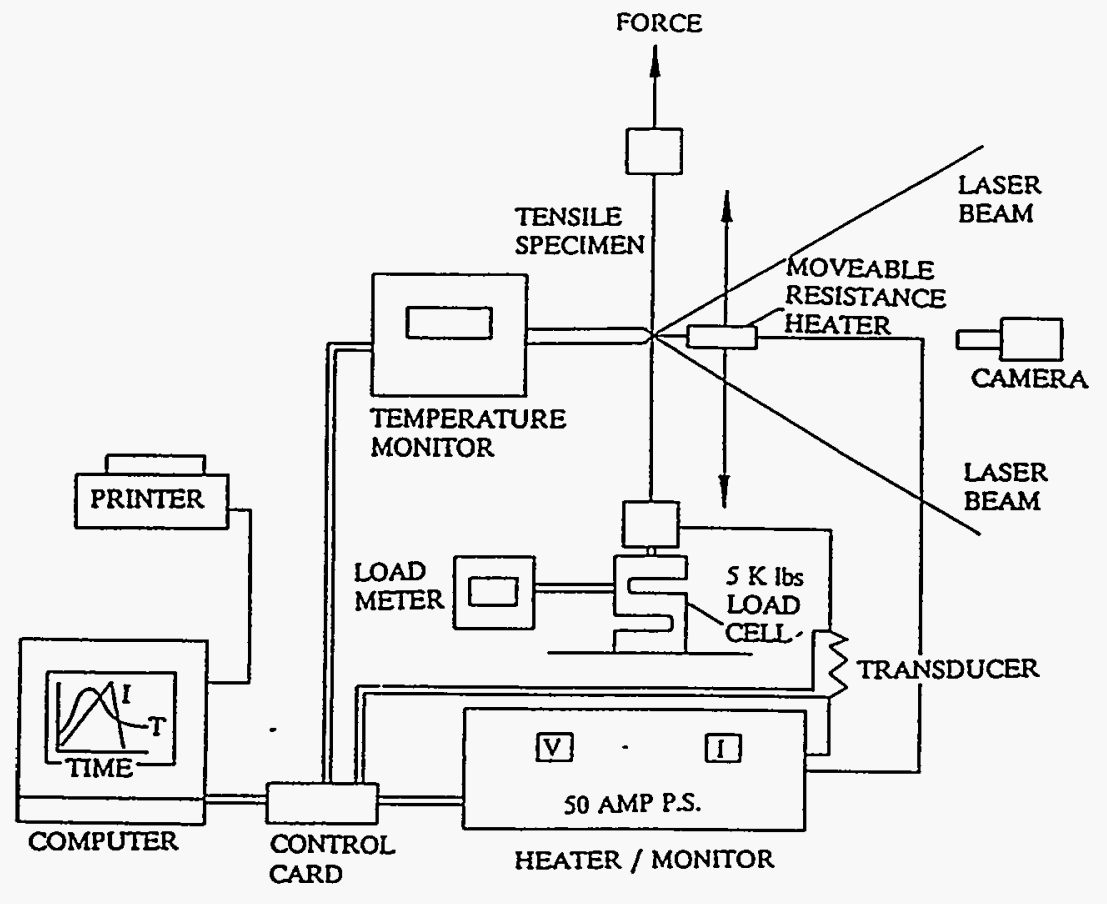

Figure 10. Schematic of the laser interferometer used in the ESPI system. The interferometer optics use a single spatial filter to minimize the number of optical elements in the set-up.

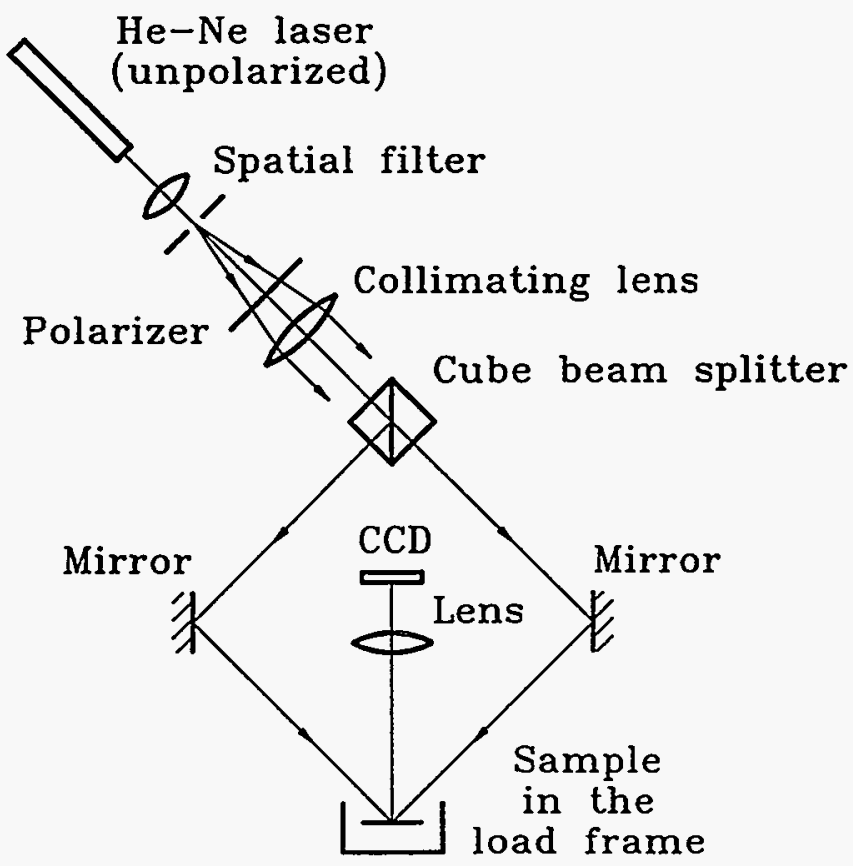


Figure 11. Schematic of the image acquisition system used in the ESPI. This was a PC based system that consisted of readily available hardware. The image subtraction and enhancement was also performed with commercially available software.

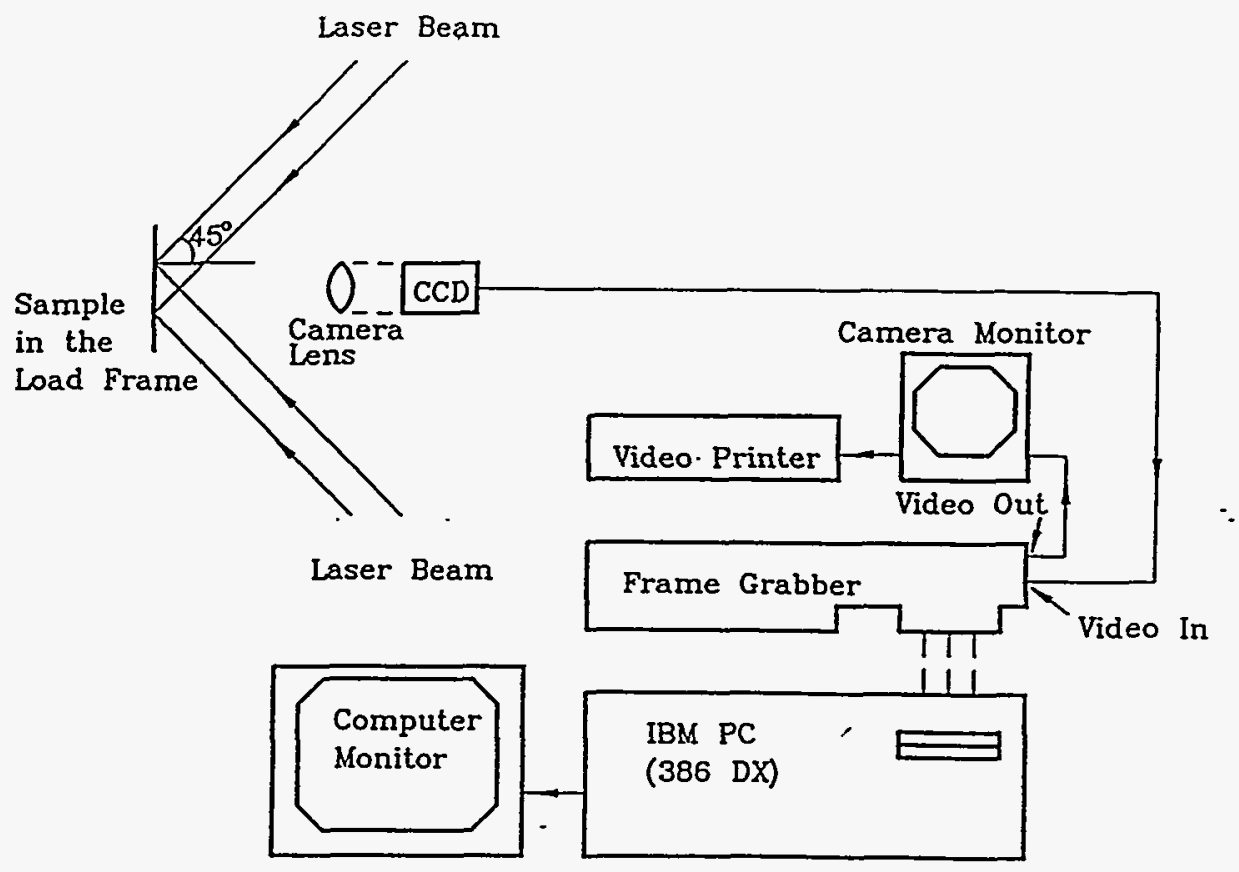

Figure 12. Spot heating cycle. The current was a square pulse lasting 3 seconds. The temperature on the rear of the specimen increased to a maximum of 73 degrees Celsius and then exponentially decayed back to room temperature in about 5 minutes. Notice the lag between the peak temperature and the end of the current pulse. This results form the thermal time constant between the front of the specimen and the rear.

TEMPERATURE AND CURRENT VS TIME

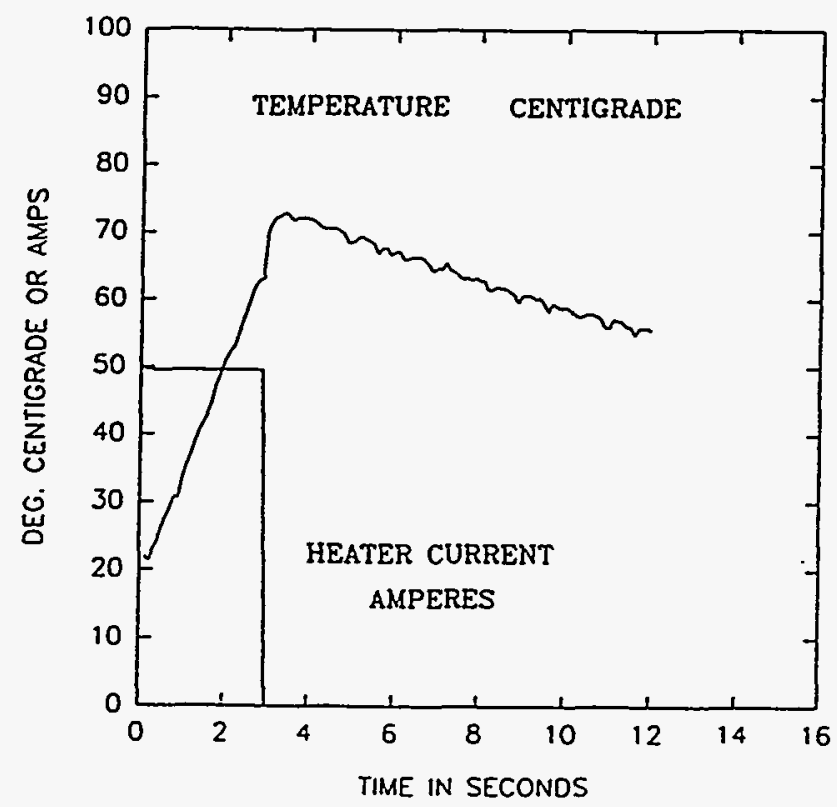


Figure 13. Fringe pattern for a specimen loaded to $80 \%$ of its yield stress. The initial load on the specimen was $36,466 \mathrm{psi}$. The stress decreased to a minimum of $35,653 \mathrm{psi}$ during the heating and then returned to the initial stress. The fringe pattern gives a displacement of 0.28 micrometers at the vertical lines which compares with 0.275 micrometers form the computer simulations.

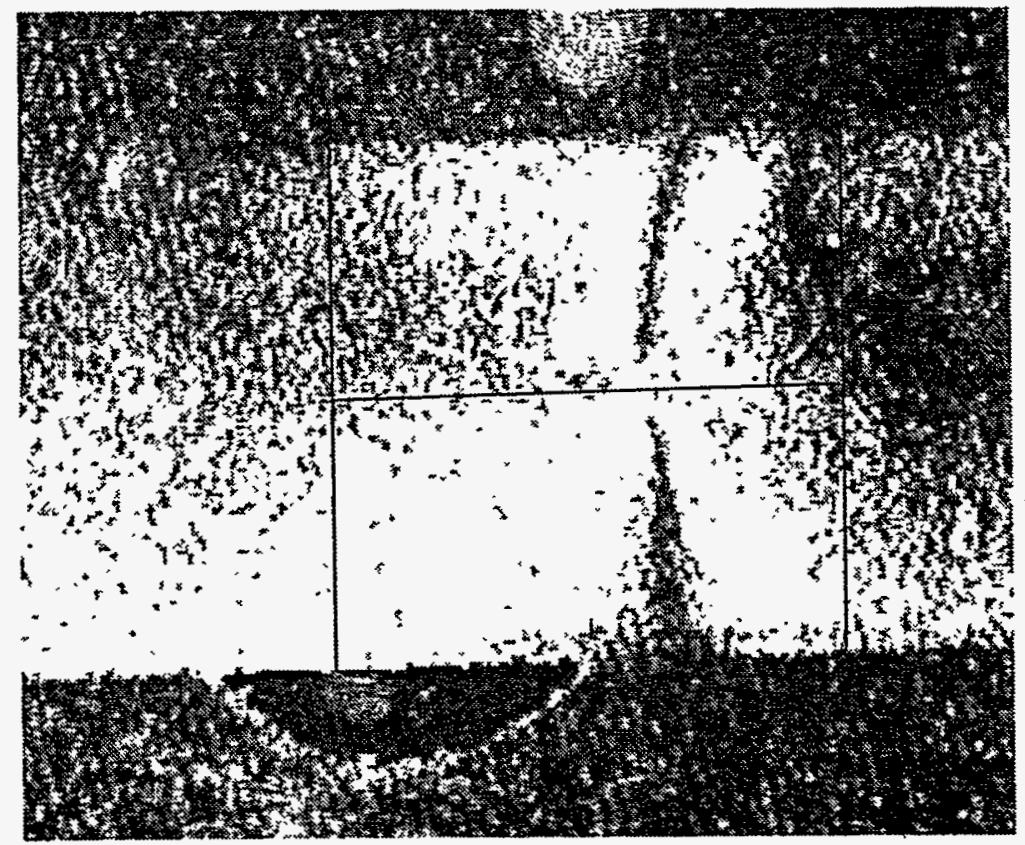

Figure 14. Fringe pattern for a specimen loaded to $90 \%$ of its yield stress. The initial load on the specimen was 41,025 psi. The stress decreased to a minimum of 40,085 psi during the heating and then returned to the initial stress. The fringe pattern gives a displacement of 1.23 micrometers at the vertical lines which compares with 1.30 micrometers form the computer simulations.

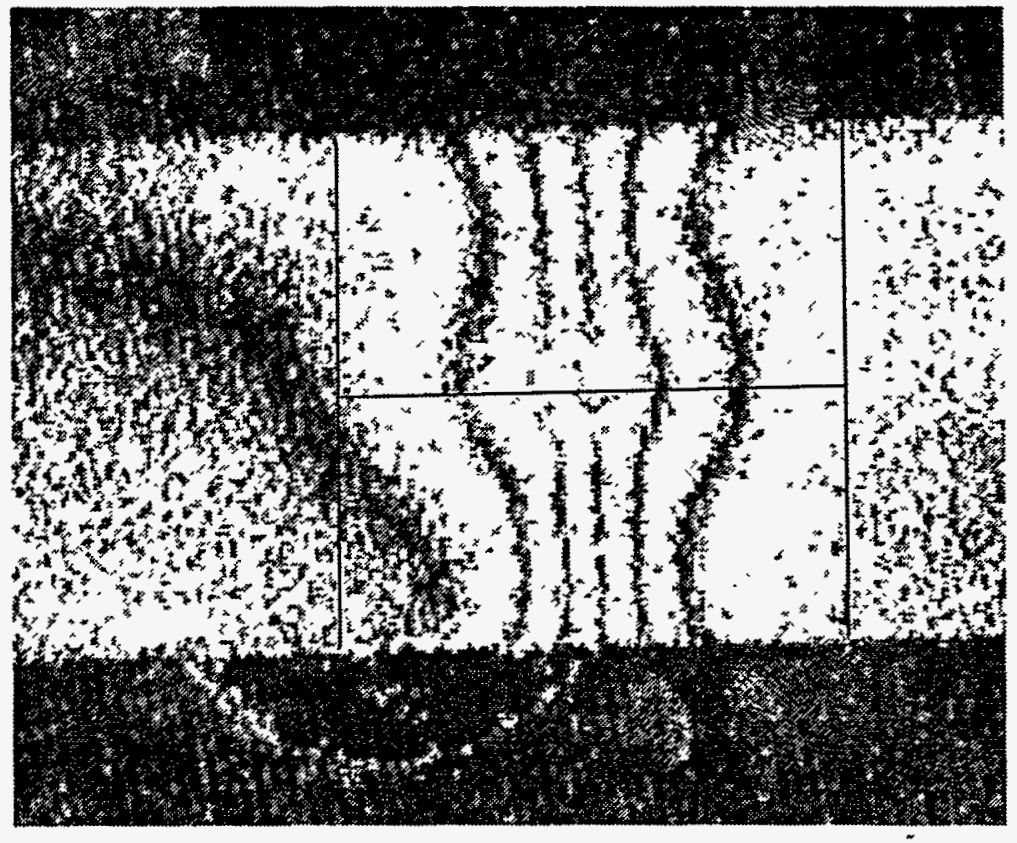


Figure 15. Fringe pattern for a specimen loaded to $98 \%$ of its yield stress. The initial load on the specimen was $44,671 \mathrm{psi}$. The stress decreased to a minimum of $42,485 \mathrm{psi}$ during the heating and then returned to the initial stress. The fringe pattem gives a displacement of 4.923 micrometers at the vertical lines which compares with 5.25 micrometers form the computer simulations.

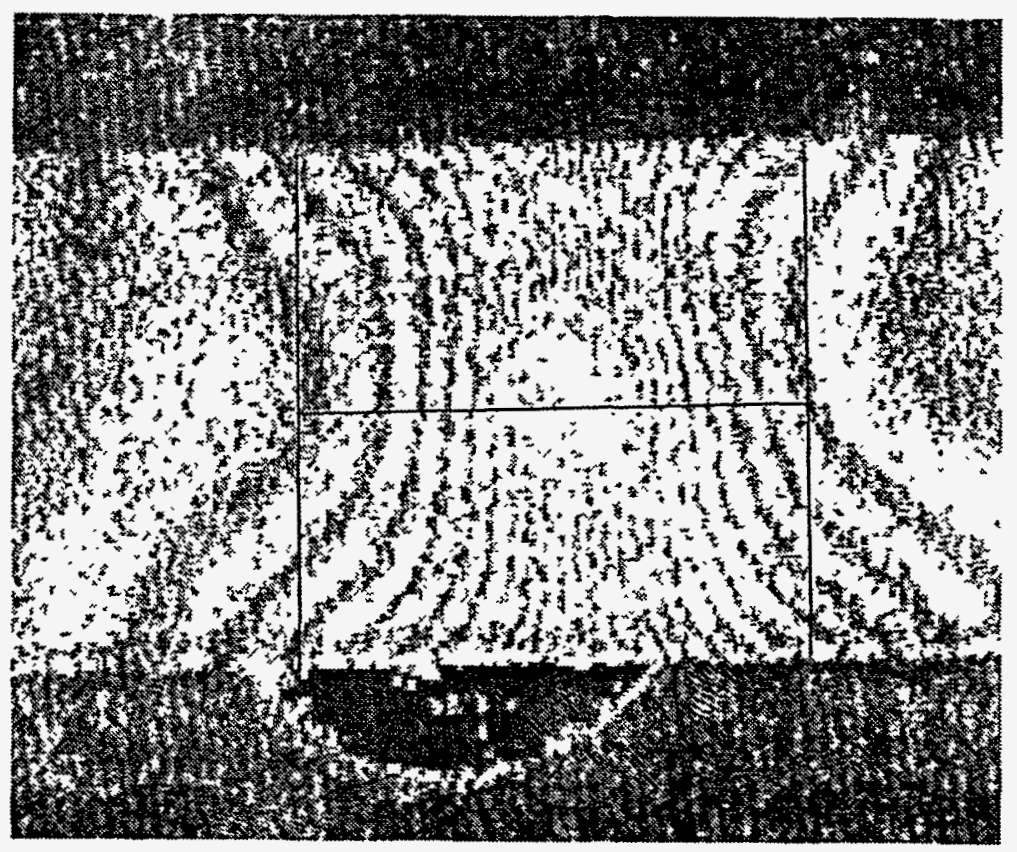

\section{REFERENCES}

1. Nickola, Wayne E., "Weld Induced Residual Stress Measurements Via the Hole-Drilling Strain Gauge Method," ASME No. 84-WAIDE-25.

2. "Determining Residual Stresses by the Hole-Drilling Strain-Gauge Method.," ASTM Standard E837-92, American Society for Testing Materials, 1992.

3. "Measurement of Residual Stresses by the Hole-Drilling Strain-Gauge Method," Technical Note TN 503-4, Measurements Group, Raleigh, NC., 1993.

4. Prevey, Paul S, X-Ray Diffraction Residual Stress Techniques In Metals Handbook (R), Ninth Ed., Vol. 10, Materials Characterization, 1985.

5. Ruud, Clayton O., "A Review of Nondestructive Methods for Residual Stress Measurement," J. of Metals, Vol 33, No. 7, July 1981, pp. $35-40$.

6. Jones, R. and C. Wykes, Holographic and Speckle Interferometry, Second Edition, pp. 159-161, Cambridge University Press, 1989.

7. Nelson, D.V. and J.J. McCrikerd, "Residual-Stress Determination Through Combined Use of Holographic Interferometry and Blind-Hole Drilling," Experimental Mechanics, pp. 371-378, December 1986.

8. Hibbitt, Karlsson \& Sorensen, Inc., ABAQUS, Version 4.9.

9. 1990 Annual Book of ASTM Standards. Section 3, Metals. Test Methods and Analytical Procedures, Section E8, page 137, ASTM, Philadelphia, PA. 